Article

\title{
Investigation on the Effect of Type of Cooling on the Properties of Aluminum Alloy during Warm/Hot Hydromechanical Deep Drawing
}

\author{
Gaoshen Cai ${ }^{1, *}$, Chuanyu $\mathrm{Wu}^{1}$ and Dongxing Zhang ${ }^{2}$ \\ 1 Faculty of Mechanical Engineering \& Automation, Zhejiang Sci-Tech University, Hangzhou 310018, China; \\ cywu@zstu.edu.cn \\ 2 Department of Mechanical \& Material Engineering, Western University, London, ON N6A 5B9, Canada; \\ zhangdx1113@gmail.com \\ * Correspondence: caigaocan@zstu.edu.cn
}

Received: 9 July 2018; Accepted: 24 August 2018; Published: 26 August 2018

\begin{abstract}
The warm sheet cylindrical deep drawing experiment of aluminum alloy was carried out and macro-mechanical properties and microstructure evolution of hydro-formed cups with different cooling medium were analyzed, which aimed to investigate the effects of different types of cooling on mechanical properties and microstructure of cylindrical cups hydro-formed by warm Hydro-mechanical Deep Drawing (HDD). Results show that, under the condition of warm hydroforming, the mechanical properties such as yield stress and ultimate strength were influenced very little by air or water cooling. Grain coarsening of these hydro-formed cups can be inhibited to a certain extent with subsequent rapid water cooling. Moreover, it shows that the processing with warm sheet hydroforming and subsequent rapid cooling of 7075-O aluminum alloy has a positive significance in maintaining the stability of macro mechanical properties and inhibiting the degradation of the microstructure of materials.
\end{abstract}

Keywords: hydro-mechanical deep drawing (HDD); mechanical property; type of cooling; microstructure

\section{Introduction}

Warm/hot sheet hydroforming has prominent advantages in improving the forming limit due to combining the advantages of both cold sheet hydroforming and warm sheet forming [1,2]. Since temperatures and fluid pressures can lead to more formability than by using cold sheet hydroforming or warm sheet forming, a warm/hot sheet hydroforming process is suitable to form the thin-wall structural parts with complex surfaces of hard-to-form materials at Room Temperature (RT), which can solve the manufacturing problems in hard shaping or hard integral shaping using conventional forming methods [3,4]. Like cold sheet hydroforming, according to the action of fluid pressure in the forming process, warm/hot sheet hydroforming is classified into two styles: passive forming and active forming, which is shown in Figure 1 [5]. When forming parts like cylindrical cups, the fluid pressure plays a supporting role in the forming process, which can be called HDD, and the passive forming style can be used. By contrast, the other style can be chosen when more active fluid pressures are needed for forming parts, which can be referred to as warm/hot sheet hydro-bulging.

Even though the plasticity of materials can be improved in a warm/hot environment, it will inevitably lead to the deterioration tendency of material properties due to microstructure variation. In addition, when encountering a reduced temperature gradient at an elevated temperature, the microstructure deterioration tendency of metal materials may be inhibited and transformed in a favorable direction [6,7]. From this, the corresponding solution to the microstructure property deterioration of materials can be determined. 


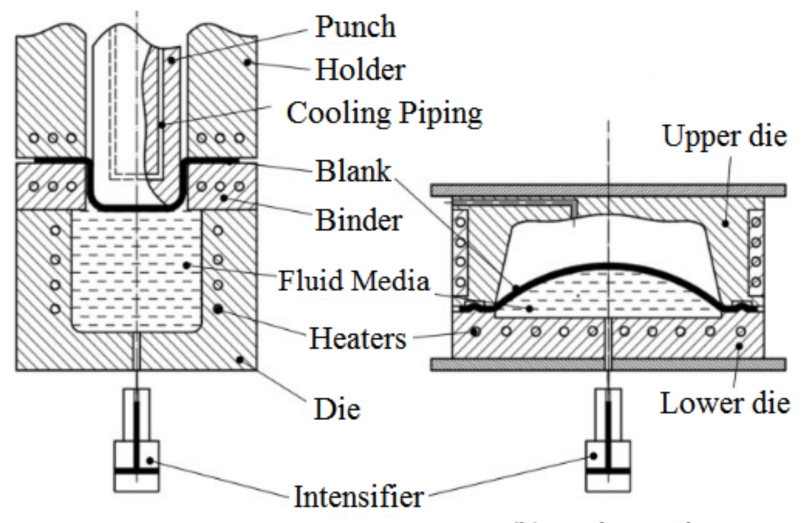

(a) Passive Style

(b) Active Style

Figure 1. Schematic of warm sheet hydroforming.

Unlike steel materials, the strengthening process of heat treatable aluminum alloys (such as 7075) is precipitation dispersion strengthening. It requires the rapid cooling of the materials from the solution temperature to room temperature rapidly and undergoes natural aging or artificial aging to strengthen its matrix due to precipitating the dispersed phase. Some relevant studies indicated [8-10] that the hydroforming technology process under a higher heat environment requires materials to have a certain strength and the forming temperature should not be too high in contrast to those with a warm or hot forming type. The hard-to-form materials, which cannot be formed using a traditional forming method, can be formed using warm/hot sheet hydroforming with subsequent rapid cooling to proceed incomplete solution strengthening. Then it can maintain the stability of macro-mechanical properties and inhibit the degradation of the microstructure of materials to a certain extent. In addition, research shows that $[5,11,12]$ assuming that the material is isotropy, stress-strain curves obtained by the bulging test have been very close to the actual flow properties of materials and can reflect the stress-strain state during the forming process. Considering that the cylindrical cups HDD experiment is a classic basic research method $[13,14]$, it was used to research the effect of the process of warm/hot sheet hydroforming with subsequent rapid cooling on mechanical properties and microstructure evolution of the cups, which were formed by warm/hot sheet HDD $[15,16]$. Meanwhile, the isotropic material was assumed in this study.

As mentioned above, using a special warm/hot sheet hydrobulging-hydromechanical deep drawing equipment, a warm/hot sheet HDD experiment of 7075-O aluminum alloy was carried out and macro mechanical properties and microstructure evolution of hydro-formed cups with different cooling medium (which represents different types of cooling) were researched, which aimed to reveal the influence rule of different types of cooling on mechanical properties and microstructure of cylindrical cups formed by warm/hot sheet hydroforming in this study. In addition, it is of great significance to further study the process characteristics of warm sheet hydroforming and inhibit the degradation of the microstructure of materials [17]. Moreover, the results can promote the application process of warm/hot sheet hydroforming technology effectively in the field of aerospace.

\section{Warm/Hot Sheet HDD Experiment}

\subsection{Experimental Material}

The HDD testing material used was an AA 7075-O aluminum alloy sheet with a thickness of $1 \mathrm{~mm}$, which was produced by Alcoa (Al-Zn-Mg-Cu series high-strength alloy). As a representative alloy used widely in the aerospace field and as a hard-to-form material at room temperature, it was selected to conduct the hydro-bulging test. Table 1 shows the chemical composition [18] and Figure 2 shows the initial microstructure of 7075-O aluminum alloy obtained using the Scanning Electron Microscope (SEM). 
Table 1. Chemical composition of 7075-O aluminum alloy (wt \%) [18].

\begin{tabular}{ccccccccccc}
\hline Composition & $\mathbf{Z n}$ & $\mathbf{M g}$ & $\mathbf{C u}$ & $\mathbf{M n}$ & $\mathbf{C r}$ & $\mathbf{F e}$ & $\mathbf{S i}$ & $\mathbf{T i}$ & others & $\mathrm{Al}$ \\
\hline Percent $(\%)$ & 5.1 & 2.1 & 1.2 & 0.3 & 0.18 & 0.5 & 0.4 & 0.2 & 0.2 & rest \\
\hline
\end{tabular}

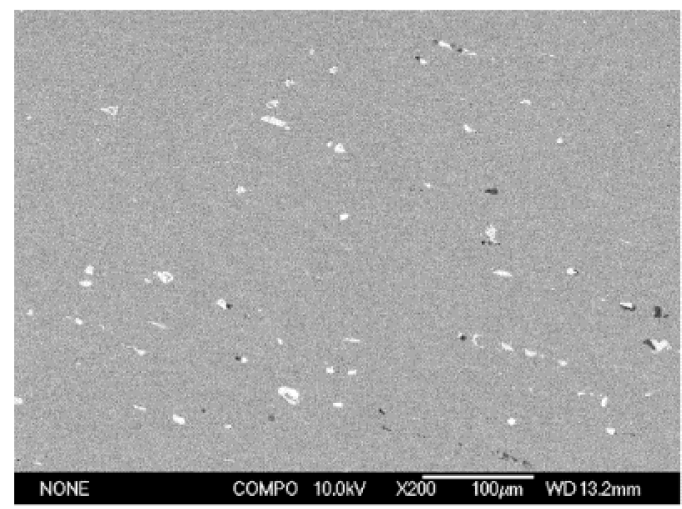

Figure 2. The initial microstructure of AA 7075-O aluminum alloy captured with the Scanning Electron Microscope (SEM).

\subsection{Test Equipment}

The HDD test equipment used was the self-development YRJ-50 machine $[6,18,19]$, by which not only can the stress-strain curve be confirmed and obtained by a warm bulging test $[19,20]$ but also a warm sheet HDD test can be carried out. It consists of a mechanical body, a detecting system, and a control system. Figure 3 shows the equipment and the setup of this HDD test.

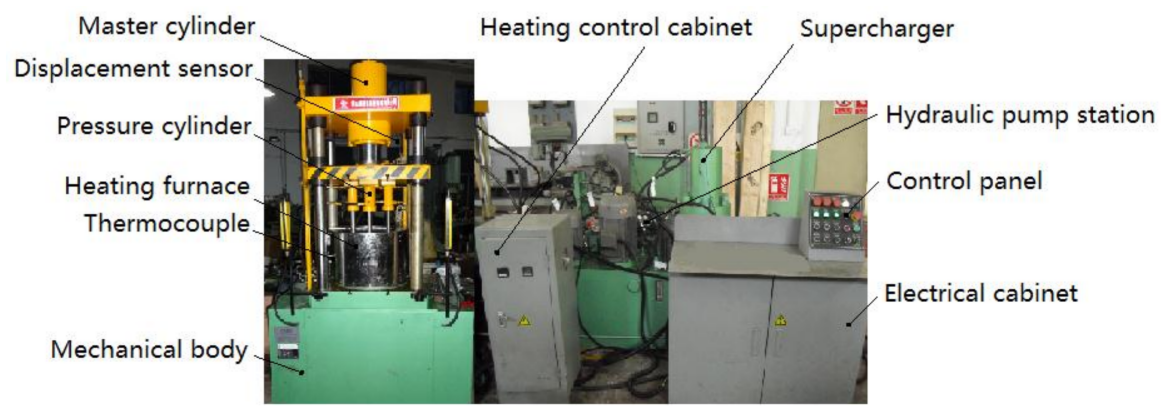

(a)

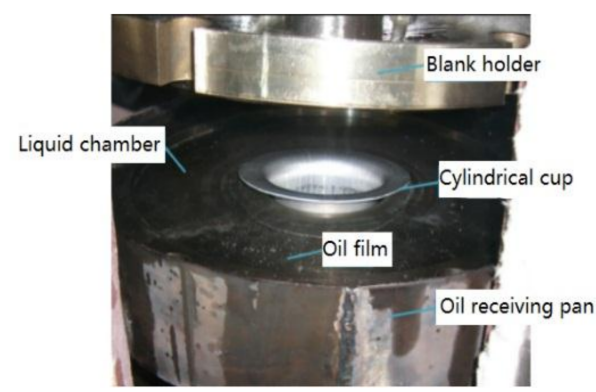

(b)

Figure 3. Equipment and setup of HDD test. (a) Warm sheet hydro-bulging-hydro-mechanical deep drawing equipment; and (b) experimental setup of warm hydro-mechanical deep drawing. 


\subsection{Experimental Results}

At RT and $210^{\circ} \mathrm{C}$, the warm sheet HDD experiment of the aluminum alloy 7075-O was carried out using the equipment mentioned above. In addition, the test conditions include: the punch diameter was $80 \mathrm{~mm}$, the punch nose radius was $10 \mathrm{~mm}$, the draw die shoulder radius was $10 \mathrm{~mm}$, the aperture of the blank holder was $81.5 \mathrm{~mm}$, the blank holder radius was $8 \mathrm{~mm}$, the inlet diameter of the liquid chamber was $85 \mathrm{~mm}$, the blank diameter was $200 \mathrm{~mm}\left(210^{\circ} \mathrm{C}\right.$ and $160 \mathrm{~mm}$ (RT), the final diameter of the cup after forming in theory was nearly $81 \mathrm{~mm}$, and the deep drawing depth was $85 \mathrm{~mm}$. As a blank holder force, liquid pressure-punch stroke curves and liquid pressure-punch stroke curves are very important influencing factors during the process of warm sheet HDD. They were designed before testing, which is shown in Figure 4a,b. After being formed, air cooling and water cooling were selected to cool the test samples to RT rapidly (the processing rate is shown in Figure 4c. Then the testing samples were obtained. Figure 5 shows the cylindrical cups hydro-formed by a warm sheet HDD at $210{ }^{\circ} \mathrm{C}$.

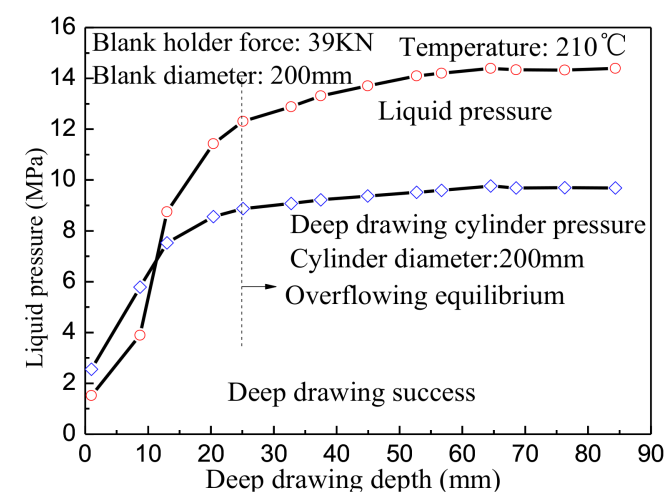

(a)

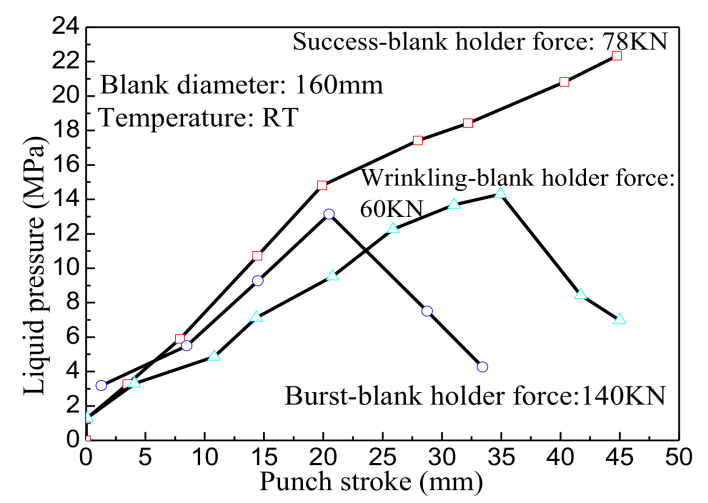

(b)

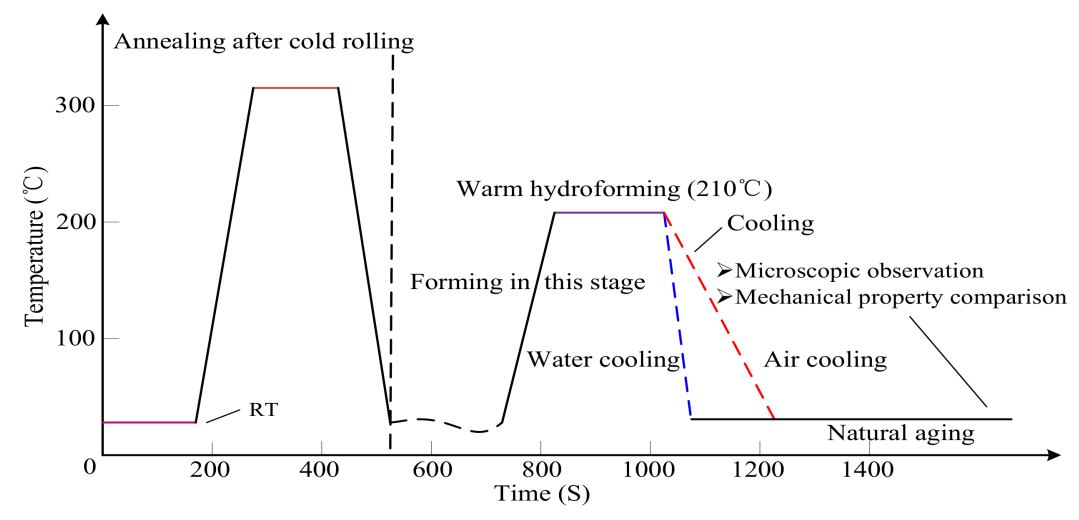

(c)

Figure 4. Experimental scheme of warm sheet HDD experiment. (a) liquid pressure-punch stroke curve and drawing force-punch stroke curve at $210{ }^{\circ} \mathrm{C}$. (b) curves of liquid pressure-punch stroke in HDD at RT; and (c) processing route of the warm sheet HDD experiment.

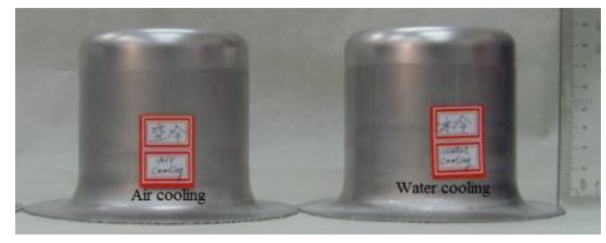

Figure 5. Cylindrical cups hydro-formed by a warm sheet HDD at $210{ }^{\circ} \mathrm{C}$. 


\section{Performance Evaluation of Aluminum Alloy Cylindrical Cups Hydro-Formed by Warm Sheet HDD}

\subsection{Evaluation Testing Scheme}

After being formed by warm/hot HDD, as the deformation of sheet materials has been accumulated, the microstructure of test sheets is in an unstable state to a certain extent. Different types of cooling (different cooling medium) represent different cooling rates in order to evaluate the macro performance of aluminum alloy cylindrical cups. The uniaxial tension samples in the straight wall of cups with the rolling direction of 0 degrees were sampled. Then, in order to evaluate their mechanical performance parameters that yield strength, tensile strength, and elongation, the uniaxial tension experiment at RT was conducted. On the other hand, with the rolling direction of 90 degrees and clipping the narrow strips with the width of $10 \mathrm{~mm}$ from the flange to the bottom of cylindrical cups, the microstructure observation samples were prepared (see Figure 6). There is a different deformation due to a different deformation area since both flange and a cup wall are the parts with large deformation during the warm/hot sheet HDD process of aluminum alloy cylindrical cups. The two positions of cups were selected to observe their microstructure variation. The performance measurement and evaluation plan of formed cylindrical cups is shown in Table 2.

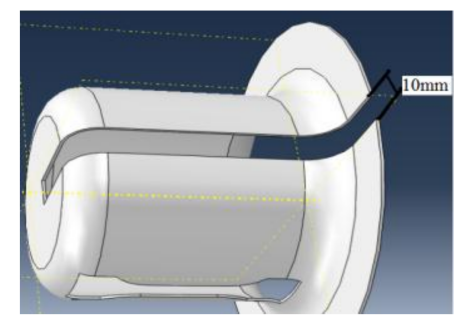

(a)

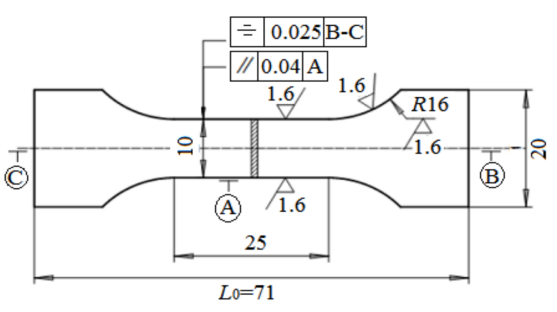

(b)

Figure 6. Schematic diagram of specimens sampling for uniaxial tension and metallographic observation. (a) sampling area for uniaxial tension and metallographic observation; and (b) sample size for uniaxial tension.

Table 2. Experimental plan for mechanical properties after warm sheet HDD.

\begin{tabular}{ccccccc}
\hline $\begin{array}{c}\text { Serial } \\
\text { Number }\end{array}$ & $\begin{array}{c}\text { Temperature } \\
\left({ }^{\circ} \mathbf{C}\right)\end{array}$ & $\begin{array}{c}\text { Maximum } \\
\text { Pressure } \\
\mathbf{( M P a )}\end{array}$ & $\begin{array}{c}\text { Sizes of Cylindrical } \\
\text { Cups } \mathbf{( m m )}\end{array}$ & Type of Cooling & $\begin{array}{c}\text { Samples of } \\
\text { Uniaxial Tension } \\
(\mathbf{m m})\end{array}$ & $\begin{array}{c}\text { Rolling } \\
\text { Direction } \\
\left({ }^{\circ}\right)\end{array}$ \\
\hline 1 & 210 & $P_{\max }=14.5$ & $t_{0}=1.0 ; \varphi=80 ; h=85$ & Water cooling & $L_{0}=71$ & 0 \\
2 & 210 & $P_{\max }=14.4$ & $t_{0}=1.0 ; \varphi=80 ; h=85$ & Water cooling & $L_{0}=71$ & 0 \\
3 & 210 & $P_{\max }=14.3$ & $t_{0}=1.0 ; \varphi=80 ; h=85$ & Water cooling & $L_{0}=71$ & 0 \\
4 & 210 & $P_{\max }=14.5$ & $t_{0}=1.0 ; \varphi=80 ; h=85$ & Air cooling & $L_{0}=71$ & $L_{0}=71$ \\
5 & 210 & $P_{\max }=14.4$ & $t_{0}=1.0 ; \varphi=80 ; h=85$ & Air cooling & $L_{0}=71$ \\
6 & 210 & $P_{\max }=14.3$ & $t_{0}=1.0 ; \varphi=80 ; h=85$ & Air cooling & 0 \\
\hline
\end{tabular}

\subsection{Effect of Type of Cooling on Mechanical Properties of Testing Samples}

In order to observe the influence of different types of cooling on mechanical properties of cylindrical cups after being formed by warm/hot sheet HDD, it was necessary to carry out the uniaxial tension test, which was conducted using the electro-hydraulic servo tension test machine Instron 8801 (in Figure 7) on the evaluation samples. During testing, two samples in each group were selected to obtain the average values, which yielded the test results. Then the following equation was used.

$$
L_{0}=5.65 \sqrt{S_{0}}
$$

where $S_{0}$ is the minimum value of the section of samples and $L_{0}$ is the initial reference length for calculating the percentage elongation, which is the sample length for uniaxial tension. This is shown 
in Figure 6b. Equation (1) describes the relation between $L_{0}$ and $S_{0}$, which means the test sample is a scaling sample conforming to the international common standard. Then the reliable and significant testing results can be insured using this method.

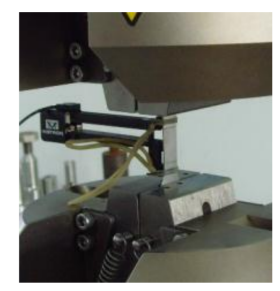

Figure 7. Experimental setup of a uniaxial tension test.

According to the performance measurement scheme, the mechanical property test results of cylindrical cups are shown in Figure 8a. It can be indicated that the yield strength is $224.18 \mathrm{MPa}$ and the ultimate strength is $260.43 \mathrm{MPa}$ under the condition of air cooling while, under the condition of water cooling, the yield strength is $228.43 \mathrm{MPa}$ and the ultimate strength is $260.66 \mathrm{MPa}$. Results show that the yield strength increases a little under water cooling compared with air cooling and the ultimate strength is nearly unchanged during either type of cooling. It indicates that different types of cooling have little effect on the mechanical properties of 7075-O aluminum alloy cylindrical cups formed using warm/hot sheet HDD. Figure $8 b$ shows the results of specific elongation after cooling using two different types of cooling. The value with air cooling is $11.83 \%$ and, with water cooling, is $11.18 \%$. Results show that the specific elongation of cylindrical cups under water cooling decreases a little more than that of air cooling. In general, though, the effect of different types of cooling on the specific elongation is not clear.

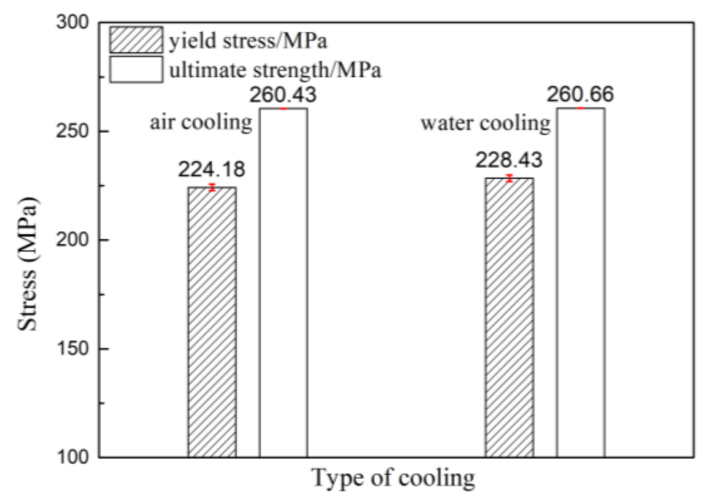

(a)

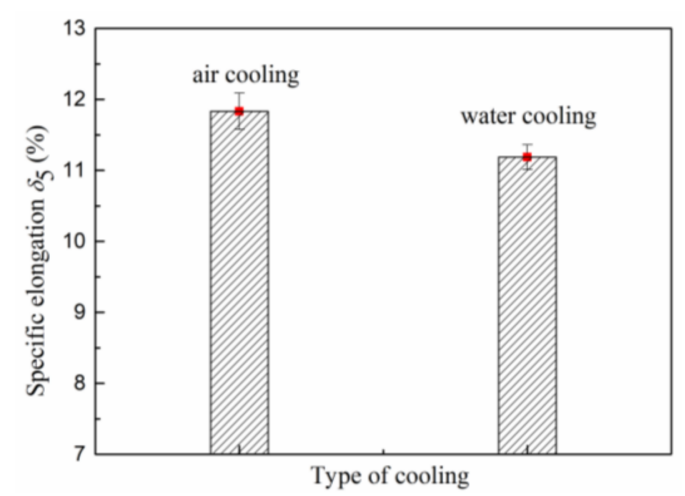

(b)

Figure 8. The influence of different types of cooling on yield stress, ultimate strength, and specific elongation. (a) Yield stress and ultimate strength; (b) Specific elongation.

In theory, the different types of cooling mainly affects the cooling rate of samples during the forming process of materials using warm/hot sheet hydroforming. Due to the result of cylindrical cups after being formed, the influence on mechanical properties may not be clear. It shows that the influence of different types of cooling on properties of aluminum alloys may be in a macro field rather than in a micro field, which is demonstrated by the experimental results described above. These results may be positively significant for maintaining the stability of macro mechanical properties of sheet metals.

\subsection{Effect of Type of Cooling on the Microstructure of Testing Samples}

The metallographic test and the Electron Back-Scattered Diffraction (EBSD) analysis on flanges and cup walls of samples after being formed using different types of cooling were carried out to 
observe the microstructure variation of cylindrical cups hydro-formed by warm/hot sheet HDD. According to GB/T 3246.1-2000, test samples and the special parts of the aluminum alloy cylindrical cups were corroded using a Keller reagent ( $1 \mathrm{~mL} \mathrm{HF}, 1.5 \mathrm{~mL} \mathrm{HCl}, 2.5 \mathrm{~mL} \mathrm{HNO}_{3}$, and $95 \mathrm{~mL} \mathrm{H}_{2} \mathrm{O}$ ). Then, using the optical microscope Axiovert 200MAT produced by the Zeiss company in Oberkochen of Germany, the samples were observed. The results are shown in Figure 9.

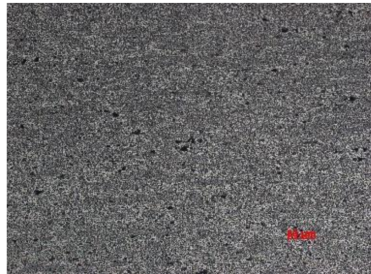

(a-1)

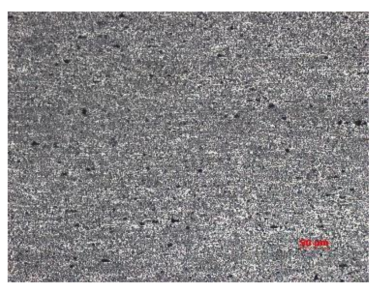

(b-1)

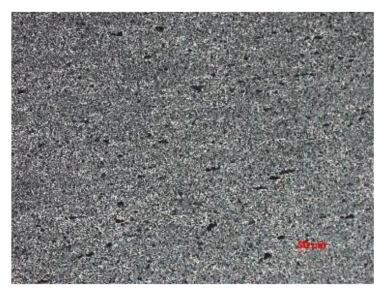

(c-1)

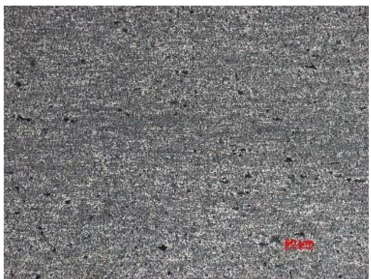

(d-1)

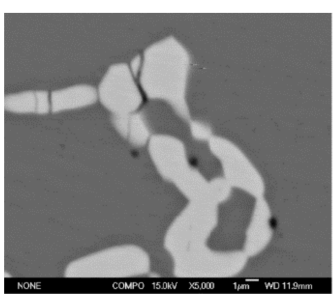

(e-1)

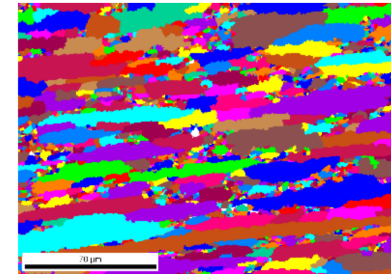

(a-2)

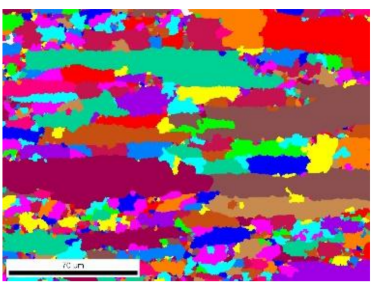

(b-2)

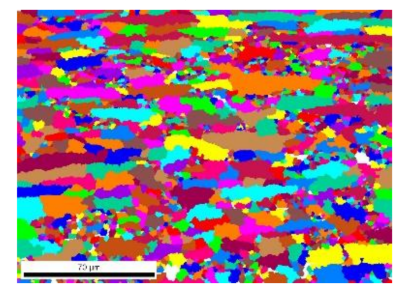

(c-2)

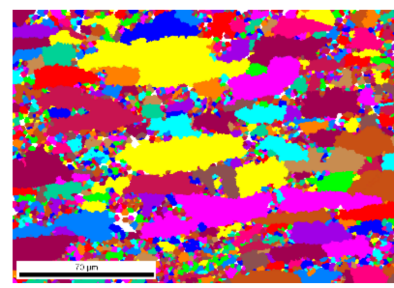

(d-2)

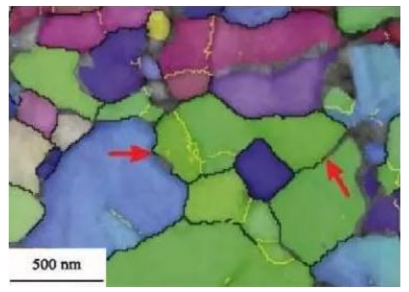

(e-2)

Figure 9. The influence of different types of cooling on microstructures. (a-1) The microstructure of flange by a metallographic test with air cooling. (a-2) The microstructure of flange by EBSD with air cooling. (b-1) The microstructure of cup wall by a metallographic test with air cooling. (b-2) The microstructure of cup wall by EBSD with air cooling. (c-1) The microstructure of flange by a metallographic test with water cooling. (c-2) The microstructure of flange by EBSD with water cooling. (d-1) The microstructure of the cup wall by a metallographic test with water cooling. (d-2) The microstructure of a cup wall by EBSD with water cooling. (e-1) The microstructure of flange by SEM with air cooling. (e-2) Orientation image of a cup wall by EBSD with water cooling. 
Studies have shown that [21-23], under the condition of a warm/elevated temperature, grain growth is mainly caused by plastic deformation and it is a dynamic recovery process. As is shown in Figure 9(a-1,c-1,e-1), after being formed using warm/hot sheet HDD, the compounds in flange of 7075-O aluminum alloy was permutated along the calendaring direction after fracture. In addition, the phase particle was precipitated in the $\alpha$ (Al) matrix, which was shown clearly in Figure 9(e-1). Figure $9(\mathrm{~b}-1, \mathrm{~d}-1)$ show that the compounds in a cylindrical cup wall further fractured, which were a stronger permutation along the deformation direction than that of flange and there was a dispersed phase in the $\alpha$ (Al) matrix. According to References [21-23], on the whole, there were more oversized second phase particles in the metallographic structure under the two types of cooling and these second phases were different in morphology including the acicular metastable phase and the punctiform stable phase [21,22]. From Figure 9(a-2) to Figure 9(e-2), they show the results of grain morphology observed using EBSD technology. Figure 9(e-2) shows the orientation image of a cup wall with water cooling from which it can be seen that, along the calendaring direction, the grain was strip, which illustrates the grain anisotropy of formed cylindrical cups in a calendaring direction and vertical direction [23].

Comparing Figure $9(a-2)$ with (b-2) and Figure $9(\mathrm{c}-2)$ with $(\mathrm{d}-2)$, it can be seen that, under the same types of cooling, the grain size in the cylindrical cup wall is larger than that of the flange. The reason is that, during the process of deep drawing, the flange was acted upon by two forces of a pressure-tensile stress (pressure stress of circumferential and thickness direction). Besides the thickness normal stress provided by hydraulic pressure, the cylindrical cup wall was also acted upon by the two forces of tensile stress.

The variation of grain size under different types of cooling are shown in Figure 10. Under the condition of air cooling, the maximum grain size in the flange was $34 \mu \mathrm{m}$, which accounts for $13.3 \%$ of the sampling area. The maximum grain size in the cylindrical cup wall was $45 \mu \mathrm{m}$, which accounts for $19.7 \%$. While under the condition of water cooling, the maximum grain size in the flange was $20 \mu \mathrm{m}$ and it accounts for $8.3 \%$ of the sampling area. The maximum grain size of the cylindrical cup wall was $37 \mu \mathrm{m}$ and it accounts for $9.4 \%$. It shows that, with the air cooling condition, there were an increase in the oversized grain. By contrast, there was grain coarsening to a certain extent with a water cooling condition, but the grain size was uniform on the whole and the grain size in the flange and cylindrical cup wall was smaller than that of air cooling. In addition, the maximum grain size appeared in the same cylindrical cup wall under both of the types of cooling while the maximum grain size under the water cooling condition was about $80 \%$ than that of air cooling. The results show that the processing warm sheet hydroforming with thereafter subsequent cooling of 7075-O aluminum alloy can inhibit the grain coarsening to a certain extent, which proves that it is of clear positive significance in maintaining the stability of macro mechanical properties and inhibiting the degradation of the microstructure of materials.
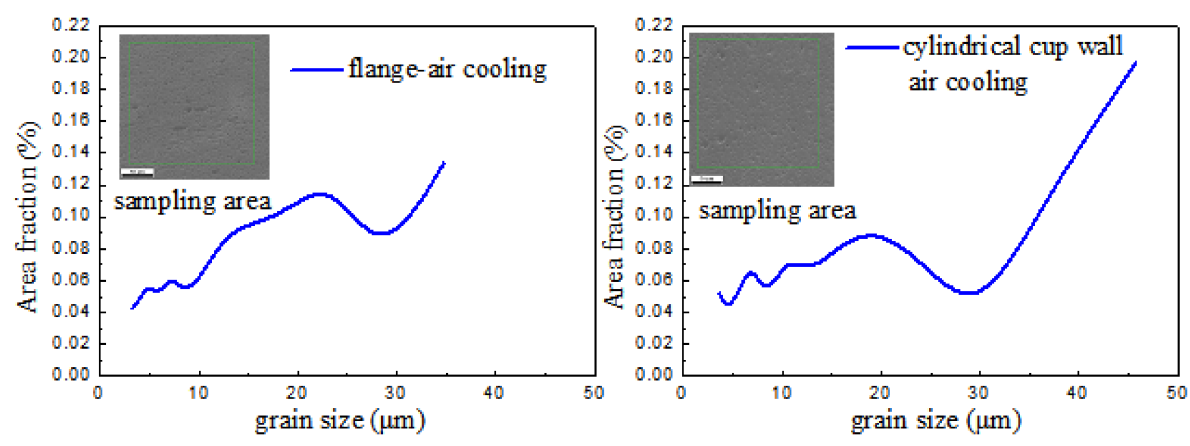

Figure 10. Cont. 

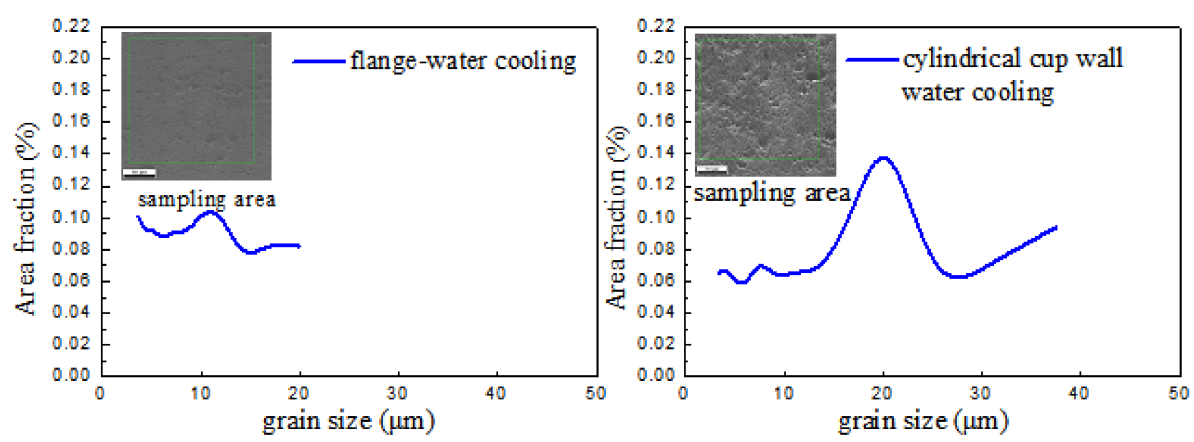

Figure 10. The influence of different types of cooling on the grain size.

\section{Conclusions}

This paper brings new experimental results concerning the effects of a type of cooling on the properties of aluminum alloy using a warm/hot sheet hydroforming process. Warm/hot sheet HDD experimentation was carried out to investigate the effects of different types of cooling on mechanical properties and microstructure evolution of cylindrical cups. The main results obtained in this study can be summarized by the following points below.

1. It shows that, under the condition of warm hydroforming, the mechanical properties of the 7075-O aluminum alloy cylindrical cups were influenced very little by different types of cooling. Compared with air cooling, there were more precipitates of the cups with water cooling, but the ultimate strength was nearly unchanged. While the yield strength increased slightly and the specific elongation tended to decrease a little under the condition of water cooling.

2. Under the condition of air cooling, the grain of the flange and the cylindrical cup wall of the formed cups were coarsened inordinately and the grain of the cylindrical cup wall was the most serious in which the maximum grain size was $45 \mu \mathrm{m}$. Alternately, under the condition of water cooling, the grain size of the flange and the cylindrical cup wall of the cups were inhibited effectively and the grain size was smaller and more uniform than that of air cooling.

3. It proves that the grain coarsening of the 7075-O aluminum alloy hydro-formed cups can be inhibited to a certain extent during warm/hot sheet hydroforming with subsequent rapid water cooling, which indicates that there is a positive significance in maintaining the stability of macro mechanical properties and inhibiting the degradation of the materials' microstructure.

Author Contributions: D.Z. analyzed the experimental data. G.C. analyzed the date and wrote this paper. C.W. proposed the ideas and reviewed the paper.

Funding: This research received no external funding.

Acknowledgments: This work was supported by the Zhejiang Provincial Natural Science Foundation (ZJNSF) with Grant No. LQ18E050010 and the Zhejiang Sci-Tech University Research Foundation with Grant No. 17022073-Y.

Conflicts of Interest: The authors declare no conflict of interest.

\section{References}

1. Lang, L.H.; Liu, B.S.; Liu, H.J.; Lyamina, E. Simulation of aluminium alloy 5A06 warm/hot hydromechanical sheet deep drawing. Mater. Sci. Forum. 2009, 623, 61-69. [CrossRef]

2. Lang, L.H.; Wang, Z.R.; Kang, D.C.; Yuan, S.J.; Zhang, S.H.; Danckert, J.; Nielsen, K.B. Hydroforming highlights: Sheet hydroforming and tube hydroforming. J. Mater. Process. Technol. 2004, 151, $165-177$. [CrossRef]

3. Liu, B.S.; Lang, L.H.; Zeng, Y.S.; Lin, J.G. Forming characteristic of sheet hydroforming under the influence of through-thickness normal stress. J. Mater. Process. Technol. 2012, 212, 1875-1884. [CrossRef] 
4. Lang, L.H.; Cai, G.S.; Liu, K.N.; Alexandrov, S.; Du, P.M.; Zheng, H. Investigation on the effect of through thickness normal stress on forming limit at elevated temperature by using modified M-K model. Int. J. Mater. Form. 2015, 8, 211-228. [CrossRef]

5. Lang, L.H.; Liu, B.S.; Li, T.; Zhao, X.N.; Zeng, Y.S. Experimental investigation on hydromechanical deep drawing of aluminum alloy with heated media. Steel Res. Int. 2012, 83, 230-237. [CrossRef]

6. Cai, G.S.; Wu, C.Y.; Gao, Z.P.; Lang, L.H.; Alexandrov, S. Research on Al-alloy sheet forming formability during warm/hot sheet hydroforming based on elliptical warm bulging test. AIP Adv. 2018, 8, 1-9. [CrossRef]

7. Lang, L.H.; Du, P.M.; Liu, B.S.; Cai, G.S.; Liu, K.N. Pressure rate controlled unified constitutive equations based on microstructure evolution for warm hydroforming. J. Alloys Compd. 2013, 574, 41-48. [CrossRef]

8. Liu, K.N.; Lang, L.H.; Cai, G.S.; Yang, X.Y.; Guo, C.; Liu, B.S. A novel approach to determine plastic hardening curves of AA7075 sheet utilizing hydraulic bulging test at elevated temperature. Int. J. Mech. Sci. 2015, 100, 328-338. [CrossRef]

9. Kaya, S.; Altan, T.; Groche, P.; Klöpsch, C. Determination of the flow stress of magnesium Az31-O sheet at elevated temperatures using the hydraulic bulge test. Int. J. Mach. Tools Manuf. 2008, 48, 550-557. [CrossRef]

10. Mahabunphachai, S.; Koc, M. Investigations on forming of aluminum 5052 and 6061 sheet alloys at warm temperatures. Mater. Des. 2010, 31, 2422-2434. [CrossRef]

11. Jeong, Y.; Gnäupel-Herold, T.; Barlat, F.; Iadicola, M.; Creuziger, A.; Lee, M.-G. Evaluation of biaxial flow stress based on elasto-viscoplastic self-consistent analysis of X-ray diffraction measurements. Int. J. Plast. 2015, 66, 103-118. [CrossRef]

12. Min, J.Y.; Stoughton, T.B.; Carsley, J.E.; Carlson, B.E.; Lin, J.P.; Gao, X.L. Accurate characterization of biaxial stress-strain response of sheet metal from bulge testing. Int. J. Plast. 2017, 94, 192-213. [CrossRef]

13. Groche, P.; Huber, R.; Doerr, J.; Schmoeckel, D. Hydromechanical deep-drawing of aluminium-alloys at Elevated Temperatures. CIRP Ann. 2002, 51, 215-218. [CrossRef]

14. Zafar, R.; Lang, L.H.; Zhang, R.J. Analysis of hydro-mechanical deep drawing and the effects of cavity pressure on quality of simultaneously formed three-layer $\mathrm{Al}$ alloy parts. Int. J. Adv. Manuf. Tech. 2015, 80, 2117-2128. [CrossRef]

15. He, D.G.; Lin, Y.C.; Chen, J.; Chen, D.D.; Huang, J.; Tang, Y.; Chen, M.S. Microstructural evolution and support vector regression model for an aged Ni-based superalloy during two-stage hot forming with stepped strain rates. Mater. Des. 2018, 154, 51-62. [CrossRef]

16. Ji, H.C.; Liu, J.P.; Wang, B.Y.; Tang, X.F.; Lin, J.G.; Huo, Y.M. Microstructure evolution and constitutive equations for the high-temperature deformation of $5 \mathrm{Cr} 21 \mathrm{Mn} 9 \mathrm{Ni} 4 \mathrm{~N}$ heat-resistant steel. J. Alloys Compd. 2017, 693, 674-687. [CrossRef]

17. Lin, Y.C.; Luo, S.C.; Yin, L.X.; Huang, J. Microstructural evolution and high temperature flow behaviors of a homogenized Sr-modified Al-Si-Mg alloy. J. Alloys Compd. 2018, 739, 590-599. [CrossRef]

18. Cai, G.S.; Zhou, X.J.; Lang, L.H.; Alexandrov, S. Research on aluminum alloy sheet thermoplastic deformation behavior based upon warm bulging test. AIP Adv. 2016, 6, 1-8. [CrossRef]

19. Cai, G.S.; Lang, L.H.; Liu, K.N.; Alexandrov, S.; Zhang, D.X.; Yang, X.Y.; Guo, C. Research on the effect of flow stress calculation on aluminum alloy sheet deformation behavior based on warm bulging test. Met. Mater. Int. 2015, 21, 365-373. [CrossRef]

20. Koç, M.; Billur, E.; Necati, Ö.N. An experimental study on the comparative assessment of hydraulic bulge test analysis methods. Mater. Des. 2011, 32, 272-281. [CrossRef]

21. Hu, J.Q. Experimental Simulation of Control Rolling Process of 7075 Aluminum Alloy. Master's Thesis, Central South University, Changsha, China, 2003.

22. Lu, J.; Song, Y.L.; Hua, L.; Zheng, K.L.; Dai, D.G. Thermal deformation behavior and processing maps of 7075 aluminum alloy sheet based on isothermal uniaxial tensile tests. J. Alloys Compd. 2018, 767, 856-869. [CrossRef]

23. Tian, W.M.; Li, S.M.; Liu, J.H.; Yu, M.; Du, Y.J. Preparation of bimodal grain size 7075 aviation aluminum alloys and their corrosion properties. Chin. J. Aeronaut. 2017, 30, 1777-1788. [CrossRef]

(C) 2018 by the authors. Licensee MDPI, Basel, Switzerland. This article is an open access article distributed under the terms and conditions of the Creative Commons Attribution (CC BY) license (http:// creativecommons.org/licenses/by/4.0/). 